\title{
Remarks on the critical Besov space and its embedding into weighted Besov-Orlicz spaces
}

\author{
by \\ Hidemitsu Wadade (Osaka)
}

\begin{abstract}
We present several continuous embeddings of the critical Besov space $B_{p}^{n / p, \rho}\left(\mathbb{R}^{n}\right)$. We first establish a Gagliardo-Nirenberg type estimate

$$
\|u\|_{\dot{B}_{q, w_{r}}^{0, \nu}} \leq C_{n}\left(\frac{1}{n-r}\right)^{\frac{1}{q}+\frac{1}{\nu}-\frac{1}{\rho}}\left(\frac{q}{r}\right)^{\frac{1}{\nu}-\frac{1}{\rho}}\|u\|_{\dot{B}_{p}^{0, \rho}}^{\frac{(n-r) p}{n q}}\|u\|_{\dot{B}_{p}^{n / p, \rho}}^{1-\frac{(n-r) p}{n q}},
$$

for $1<p \leq q<\infty, 1 \leq \nu<\rho \leq \infty$ and the weight function $w_{r}(x)=1 /|x|^{r}$ with $0<r<n$. Next, we prove the corresponding Trudinger type estimate, and obtain it in terms of the embedding $B_{p}^{n / p, \rho}\left(\mathbb{R}^{n}\right) \hookrightarrow B_{\Phi_{0}, w_{r}}^{0, \nu}\left(\mathbb{R}^{n}\right)$, where the function $\Phi_{0}$ of the weighted Besov-Orlicz space $B_{\Phi_{0}, w_{r}}^{0, \nu}\left(\mathbb{R}^{n}\right)$ is a Young function of the exponential type. Another point of interest is to embed $B_{p}^{n / p, \rho}\left(\mathbb{R}^{n}\right)$ into the weighted Besov space $B_{p, w_{n}}^{0, \rho}\left(\mathbb{R}^{n}\right)$ with the critical weight $w_{n}(x)=1 /|x|^{n}$; more precisely, we prove $B_{p}^{n / p, \rho}\left(\mathbb{R}^{n}\right) \hookrightarrow B_{p, W_{s}}^{0, \rho}\left(\mathbb{R}^{n}\right)$ with the weight $W_{s}(x)=\frac{1}{|x|^{n}[\log (e+1 /|x|)]^{3}}$ for any $s>1$.
\end{abstract}

1. Introduction and main results. The main purpose of this paper is to investigate the properties of the critical Besov space in terms of continuous embeddings into weighted Besov or Besov-Orlicz spaces. Firstly, we should recall the Gagliardo-Nirenberg type inequalities on the fractional Sobolev space with the critical differential order, $H_{p}^{n / p}\left(\mathbb{R}^{n}\right)$, where $n \in \mathbb{N}$ and $1<p$ $<\infty$. The Sobolev embedding theorem states that $H_{p}^{n / p}\left(\mathbb{R}^{n}\right)$ can be embedded into $L_{q}\left(\mathbb{R}^{n}\right)$ for all $q$ with $p \leq q<\infty$, but not into $L_{\infty}\left(\mathbb{R}^{n}\right)$. Ozawa $[\mathrm{Oz}$ gave a precise estimate for this embedding:

$$
\|u\|_{L_{q}} \leq C_{n, p} q^{1 / p^{\prime}}\|u\|_{L_{p}}^{p / q}\left\|(-\Delta)^{n / 2 p} u\right\|_{L_{p}}^{1-p / q}
$$

holds for all $u \in H_{p}^{n / p}\left(\mathbb{R}^{n}\right)$ and $p \leq q<\infty$, where $p^{\prime}:=p /(p-1)$ denotes the Hölder conjugate exponent of $p$. Furthermore, $C_{n, p}$ indicates that the constant depends only on $n$ and $p$, a convention we shall adopt throughout this paper. The inequality (1.1) was originally obtained by Ogawa $\mathrm{Og}$

2010 Mathematics Subject Classification: Primary 46E35; Secondary 26D15.

Key words and phrases: Sobolev embedding theorem, Gagliardo-Nirenberg type inequality, Trudinger type inequality, Besov-Orlicz space. 
and Ogawa-Ozawa $\mathrm{OgOz}$ in the case $p=2$, i.e., for $H_{2}^{n / 2}\left(\mathbb{R}^{n}\right)$, and then (1.1) was generalized further in several ways. We refer to Nagayasu-Wadade [NW, who established a generalization of (1.1) with a weighted Lebesgue norm of the following type:

$$
\|u\|_{L_{q, w_{r}}} \leq C_{n, p}\left(\frac{1}{n-r}\right)^{\frac{1}{q}+\frac{1}{p^{\prime}}} q^{\frac{1}{p^{\prime}}}\|u\|_{L_{p}}^{\frac{(n-r) p}{n q}}\left\|(-\Delta)^{\frac{n}{2 p}} u\right\|_{L_{p}}^{1-\frac{(n-r) p}{n q}}
$$

for all $u \in H_{p}^{n / p}\left(\mathbb{R}^{n}\right), 0 \leq r<n$ and $\tilde{p} \leq q<\infty$, where $\tilde{p} \in(p, \infty)$ only depends on $n$ and $p$, and the weight function $w_{r}$ is the homogeneous function

$$
w_{r}(x):=1 /|x|^{r} \quad \text { for } x \in \mathbb{R}^{n} \backslash\{0\} .
$$

In [NW], the authors concentrated on the investigation of the growth order in $q$ as $q \rightarrow \infty$, and they proved the inequality $(1.2)$ for $q \geq \tilde{p}$, where the constant $\tilde{p}$ is chosen suitably.

Note that $L_{q, w_{r}}$ on the left-hand side of 1.2 represents the weighted Lebesgue space, and in general, for a positive measurable function $w$, we define the weighted Lebesgue space $L_{p, w}\left(\mathbb{R}^{n}\right)=L_{p}\left(\mathbb{R}^{n} ; w(x) d x\right)$ endowed with the norm

$$
\begin{aligned}
\|u\|_{L_{p, w}} & :=\left(\int_{\mathbb{R}^{n}}|u(x)|^{p} w(x) d x\right)^{1 / p} \text { for } 1 \leq p<\infty, \\
\|u\|_{L_{\infty}, w} & :=\|u\|_{L_{\infty}} .
\end{aligned}
$$

Recall that the particular weight $w_{r}$ in $(1.3)$ belongs to the class of Muckenhoupt weights, which was originally defined in Muckenhoupt $[\mathrm{M}$. Moreover, the special case $r=0$ in 1.2 coincides with 1.1 with the same growth order $q^{1 / p^{\prime}}$ as $q \rightarrow \infty$.

For another way to generalize [1.1), we refer to Wadade [W]. In [W], the author obtained the following Gagliardo-Nirenberg type inequality on the critical Besov space:

$$
\|u\|_{L_{q}} \leq C_{n, p} q^{1 / \rho^{\prime}}\|u\|_{L_{p}}^{p / q}\|u\|_{\dot{B}_{p}^{n / p, \rho}}^{1-p / q}
$$

for all $u \in\left(L_{p} \cap \dot{B}_{p}^{n / p, \rho}\right)\left(\mathbb{R}^{n}\right), 1 \leq p \leq q<\infty$ and $1<\rho \leq \infty$. The inequality 1.5 can also be regarded as a generalization of 1.1 . Indeed, by noting the embedding $\dot{H}_{p}^{n / p}\left(\mathbb{R}^{n}\right) \hookrightarrow \dot{B}_{p}^{n / p, p}\left(\mathbb{R}^{n}\right)$ if $2 \leq p<\infty$, and taking $p=\rho$ in (1.5), we have (1.1) immediately in the case $2 \leq p<\infty$. We refer to Bergh-Löfström [BL] and Triebel [T1, T2, T3] for the relationship between Sobolev and Besov spaces, and their detailed properties as function spaces. Additionally, an estimate similar to (1.5) was also given in [W]:

$$
\|u\|_{\dot{B}_{q}^{0, \nu}} \leq C_{n} q^{1 / \nu-1 / \rho}\|u\|_{\dot{B}_{p}^{0, \nu}}^{p / q}\|u\|_{\dot{B}_{p}^{n / p, \rho}}^{1-p / q}
$$

for all $u \in\left(\dot{B}_{p}^{0, \nu} \cap \dot{B}_{p}^{n / p, \rho}\right)\left(\mathbb{R}^{n}\right), 1 \leq p \leq q<\infty$ and $1 \leq \nu<\rho \leq \infty$. 
Keeping the above results in mind, we shall prove a Gagliardo-Nirenberg type interpolation inequality from the critical Besov space into a weighted Besov space, which is a generalization of the inequalities $(1.2)$ and (1.6). The definition of weighted Besov spaces will be given in (2.2) and (2.3) in Section 2. We refer to Bui [B1, B2, B3] and Triebel [T3] for detailed information about weighted Besov and weighted Triebel-Lizorkin spaces. Our first theorem now reads:

Theorem 1.1. Let $n \in \mathbb{N}$. Then there exists $C_{n}>0$ such that

$\|u\|_{\dot{B}_{q, w_{r}}^{0, \nu}} \leq C_{n}\left(\frac{1}{r}\right)^{\frac{1}{\nu}-\frac{1}{\rho_{1}}}\left(\frac{1}{n-r}\right)^{\frac{1}{q}+\frac{1}{\nu}-\frac{1}{\rho_{2}}} q^{\frac{1}{\nu}-\frac{1}{\max \left\{\rho_{1}, \rho_{2}\right\}}}\|u\|_{\dot{B}_{p}^{0, \rho_{1}}}^{\frac{(n-r) p}{n q}}\|u\|_{\dot{B}_{p}^{n / p, \rho_{2}}}^{1-\frac{(n-r) p}{n q}}$

for all $u \in\left(\dot{B}_{p}^{0, \rho_{1}} \cap \dot{B}_{p}^{n / p, \rho_{2}}\right)\left(\mathbb{R}^{n}\right), 1<p \leq q<\infty, 0<r<n, 1 \leq \nu \leq$ $\min \left\{\rho_{1}, \rho_{2}\right\} \leq \infty$, where the weight function $w_{r}$ is as in 1.3 .

REMARK. (i) The inequality (1.7) cannot hold in the limiting cases $r=0$ and $q=\infty$ in general. However, as expected from the powers in the constants of the right-hand side, the special cases $\nu=\rho_{1}$ and $\nu=\max \left\{\rho_{1}, \rho_{2}\right\}$ enable us to take $r=0$ and $q=\infty$, respectively. On the other hand, we cannot put $r=n$ all the time when the weight function becomes $1 /|x|^{n}$, and this limiting case will be the next target in our consideration.

(ii) The particular case with $\nu=\rho_{1}$ and $r=0$ coincides with (1.6) where the growth order as $q \rightarrow \infty$ becomes $q^{1 / \nu-1 / \rho_{2}}$. Furthermore, take $\nu=1$ and $2 \leq p=\rho_{1}=\rho_{2}<\infty$ in (1.7), and note the well-known embeddings

$$
\begin{cases}\|u\|_{L_{p, w}} \leq\|u\|_{\dot{B}_{p, w}^{0,1}} & \text { for all } 1 \leq p \leq \infty, \\ \|u\|_{\dot{B}_{p}^{s, p}} \leq C_{n, s, p}\|u\|_{\dot{H}_{p}^{s}} & \text { for all } s \in \mathbb{R} \text { and } 2 \leq p<\infty .\end{cases}
$$

Then the inequality (1.2) except for $r=0$ follows from 1.7$)$ and 1.8$)$ with the same growth orders as $r \uparrow n$ and $q \rightarrow \infty$.

Observe that in the limiting case $r=n$ the inequality (1.7) fails because the critical weight $1 /|x|^{n}$ is not integrable near the origin. Keeping this in mind, the next goal is to establish the embedding of the critical Besov space into a weighted Besov space, where the weight function is almost critical, namely, we take the weight as $\frac{1}{|x|^{n}(\log (1 /|x|))^{s}}$ near the origin with $s>1$. Our second result now reads:

Theorem 1.2. Let $n \in \mathbb{N}, 1<p<\infty$ and $1 \leq \rho \leq \infty$.

(i) (Subcritical weight case) The following continuous embedding holds: $B_{p}^{r / p, \rho}\left(\mathbb{R}^{n}\right) \hookrightarrow B_{p, w_{r}}^{0, \rho}\left(\mathbb{R}^{n}\right)$, where $w_{r}(x):=1 /|x|^{r}$ with $0 \leq r<n$. 
Furthermore, we have the estimate

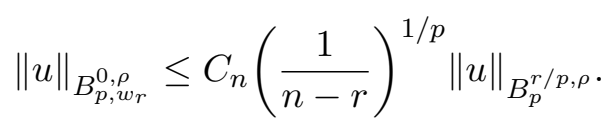

(ii) (Critical weight case) The following continuous embedding holds: $B_{p}^{n / p, \rho}\left(\mathbb{R}^{n}\right) \hookrightarrow B_{p, W_{s}}^{0, \rho}\left(\mathbb{R}^{n}\right)$, where $W_{s}(x):=\frac{1}{|x|^{n}[\log (e+1 /|x|)]^{s}}$ with $s>1$. Furthermore, we have the estimate

$$
\|u\|_{B_{p, W s}^{0, \rho}} \leq C_{n, p, s}\|u\|_{B_{p}^{n / p, \rho}}
$$

REMARK. (i) There are more general results on such embeddings in the case of Besov and Triebel-Lizorkin spaces including the Sobolev scale (cf. Haroske-Skrzypczak [HS] and Kühn-Leopold-Sickel-Skrzypczak [KLSS1, KLSS2]), but restricted to Muckenhoupt weights or so-called admissible weights the former weights may have a local singularity, while the latter are a class of smooth functions. We emphasize that these classes of weight functions do not cover the limiting situation of Theorem 1.2(ii). Indeed, it is well known that the weight $W_{s}$ is not even a Muckenhoupt weight.

(ii) The assertion of Theorem 1.2(ii) might inspire us to consider the continuous embedding from $B_{p}^{n / p, \rho}\left(\mathbb{R}^{n}\right)$ into $B_{p, \mathcal{W}_{s}}^{0, \rho}\left(\mathbb{R}^{n}\right)$, where $\mathcal{W}_{s}$ is the weight function of the double logarithmic type:

$$
\mathcal{W}_{s}(x) \simeq \frac{1}{|x|^{n}\left(\log \frac{1}{|x|}\right)\left[\log \left(\log \frac{1}{|x|}\right)\right]^{s}} \quad \text { for }|x| \ll 1 \text { with } s>1 .
$$

However, we do not explore the case of multiple logarithmic weights in this article; this will be studied in the forthcoming paper.

As another kind of a critical Sobolev embedding, we next consider a Trudinger type inequality. As already stated, the Gagliardo-Nirenberg type inequality 1.1 was obtained in $\mathrm{Oz}$. with the optimal growth order $q^{1 / p^{\prime}}$ as $q \rightarrow \infty$. In the same paper, the author also showed that (1.1) implies the following Trudinger type embedding:

$$
\left\{\begin{array}{l}
H_{p}^{n / p}\left(\mathbb{R}^{n}\right) \hookrightarrow L_{\Phi_{1}}\left(\mathbb{R}^{n}\right), \\
\Phi_{1}(t):=\exp \left(t^{p^{\prime}}\right)-\sum_{k=0}^{k_{1}-1} \frac{t^{p^{\prime} k}}{k !}, \quad k_{1}:=\min \left\{k \in \mathbb{N}: p^{\prime} k \geq p\right\},
\end{array}\right.
$$

where $1<p<\infty$ and $L_{\Phi_{1}}\left(\mathbb{R}^{n}\right)$ denotes the usual Orlicz space with the Young function $\Phi_{1}$. For the precise definition of the Orlicz space, see 2.5) and (2.6) in Section 2, where the weighted Orlicz space $L_{\Phi, w}\left(\mathbb{R}^{n}\right)$ will be introduced, and note that $L_{\Phi}\left(\mathbb{R}^{n}\right)=L_{\Phi, 1}\left(\mathbb{R}^{n}\right)$. We refer to Rao-Ren [RaRe] for abundant information about Orlicz spaces defined on general measure spaces. 
The same procedure which shows that the inequality (1.1) yields the embedding (1.9) can also be applied to the inequalities $(1.2)$ and $(1.5)$. Indeed, in Nagayasu-Wadade [NW] the authors derived from (1.2) the Trudinger type embedding

$$
\left\{\begin{array}{l}
H_{p}^{n / p}\left(\mathbb{R}^{n}\right) \hookrightarrow L_{\Phi_{2}, w_{r}}\left(\mathbb{R}^{n}\right), \\
\Phi_{2}(t):=\exp \left(t^{p^{\prime}}\right)-\sum_{k=0}^{k_{2}-1} \frac{t^{p^{\prime} k}}{k !}, \quad k_{2}:=\min \left\{k \in \mathbb{N}: p^{\prime} k \geq \tilde{p}\right\},
\end{array}\right.
$$

where $1<p<\infty, 0 \leq r<n$ and $\tilde{p}$ depends only on $n$ and $p$. Note that the embedding (1.10) generalizes (1.9) since the special case $r=0$ in 1.10 corresponds to $(1.9)$. Furthermore, the inequality 1.5 obtained in Wadade W] implies the embedding

$$
\left\{\begin{array}{l}
\left(L_{p} \cap \dot{B}_{p}^{n / p, \rho}\right)\left(\mathbb{R}^{n}\right) \underset{L_{\Phi_{3}}\left(\mathbb{R}^{n}\right),}{k_{3}-1} \Phi_{3}(t):=\exp \left(t^{\rho^{\prime}}\right)-\sum_{k=0}^{\rho^{\prime} k} \frac{k_{3}}{k !}, \quad k_{3}=\min \left\{k \in \mathbb{N}: \rho^{\prime} k \geq p\right\},
\end{array}\right.
$$

for $1 \leq p<\infty$ and $1<\rho \leq \infty$. Note that the embedding (1.11) is also a generalization of (1.9). Indeed, we can obtain $(1.9)$ in the case $2 \leq p<\infty$ by taking $p=\rho$ in (1.11) and using the embedding $\dot{H}_{p}^{n / p}\left(\mathbb{R}^{n}\right) \hookrightarrow \dot{B}_{p}^{n / p, p}\left(\mathbb{R}^{n}\right)$ for $2 \leq p<\infty$.

As seen in the above observations, the Gagliardo-Nirenberg type inequality in the critical space provides the corresponding Trudinger type embedding in general. Keeping this in mind, our final goal in the present paper is to establish a Trudinger type estimate corresponding to the inequality 1.7 in Theorem 1.1. However, the method to get embeddings 1.9), 1.10 and (1.11) through the Gagliardo-Nirenberg type inequalities can no longer work for (1.7) since the norm on the left-hand side of $(1.7)$ is a weighted Besov norm, while the inequalities (1.1), (1.2) and (1.5) have Lebesgue type norms on the left-hand side which are all formed by direct integration of functions. Therefore, we shall prove the expected Trudinger type estimate without making use of (1.7) by calculating the corresponding Besov-Orlicz norm directly. For simplicity, we restrict our considerations to the case $\rho_{1}=\rho_{2}$ in (1.7), which then becomes

$$
\|u\|_{\dot{B}_{q, w_{r}}^{0, \nu}} \leq C_{n, r} q^{\frac{1}{\nu}-\frac{1}{\rho}}\|u\|_{\dot{B}_{p}^{0, \rho}}^{\frac{(n-r) p}{n q}}\|u\|_{\dot{B}_{p}^{n / p, \rho}}^{1-\frac{(n-r) p}{n q}}
$$

for $1<p \leq q<\infty, 0<r<n$ and $1 \leq \nu<\rho \leq \infty$. To construct the Trudinger type estimate corresponding to 1.12 , it is natural to introduce weighted Besov-Orlicz spaces with an exponential type Young function (see the definition (2.4) in Section 2). Thus our last theorem now reads: 
Theorem 1.3. Let $n \in \mathbb{N}, 1<p<\infty, 0 \leq r<n, 1 \leq \nu<\rho \leq \infty$ with $(\nu, \rho) \neq(1, \infty)$ and $0<\delta \leq \mu-1$, where

$$
\mu:= \begin{cases}\frac{\rho \nu}{\rho-\nu} & \text { if } \rho<\infty, \\ \nu & \text { if } \rho=\infty .\end{cases}
$$

Then the following continuous embedding holds:

$$
\left\{\begin{array}{l}
B_{p}^{n / p, \rho}\left(\mathbb{R}^{n}\right) \hookrightarrow B_{\Phi_{0}, w_{r}}^{0, \nu}\left(\mathbb{R}^{n}\right), \\
\Phi_{0}(t):=\exp \left(t^{\mu-\delta}\right)-\sum_{k=0}^{k_{0}-1} \frac{t^{(\mu-\delta) k}}{k !}, \quad k_{0}:=\min \{k \in \mathbb{N}:(\mu-\delta) k \geq p\} .
\end{array}\right.
$$

Furthermore, we have the estimate

$$
\|u\|_{B_{\Phi_{0}, w_{r}}^{0, \nu}} \leq C_{n, \delta}\left(\frac{1}{n-r}\right)^{2 /(\mu-\delta)}\|u\|_{B_{p}^{n / p, \rho}} .
$$

REMARK. Since the growth order as $q \rightarrow \infty$ in $(1.12)$ is $q^{1 / \nu-1 / \rho}$, one would expect that the condition $\delta>0$ can be removed. However, a technical reason forces us to assume $\delta>0$ in the proof. Furthermore, the condition $\delta \leq \mu-1$, that is, $\mu-\delta \geq 1$ guarantees that $\Phi_{0}$ is a Young function. Indeed, if $\mu-\delta<1$, then $\Phi_{0}$ is no longer a Young function, which implies the weighted Besov-Orlicz space is not necessarily a normed space.

Let us describe the organization of this article. Section 2 is devoted to defining weighted function spaces and preparing lemmas for the proof of the main theorems; the theorems are proved in Section 3.

2. Preliminaries. In this section, we first give the definition of weighted Besov spaces, and then prove several lemmas. Let $\varphi$ be a non-negative function in the Schwartz class $\mathcal{S}\left(\mathbb{R}^{n}\right)$ such that $\operatorname{supp} \varphi=\{1 / 2 \leq|x| \leq 2\}$, $\varphi(x)>0$ for all $x$ with $1 / 2<|x|<2$ and $\sum_{j=-\infty}^{\infty} \varphi\left(2^{-j} x\right)=1$ if $x \neq 0$. It is well known that such a function exists (see, for instance, Bergh-Löfström $[\mathrm{BL}])$. Moreover, we define $\mathcal{S}\left(\mathbb{R}^{n}\right)$ functions $\varphi_{j}$ for $j \in \mathbb{Z}$ and $\psi$ as follows:

$$
\hat{\varphi}_{j}(x)=\varphi\left(2^{-j} x\right) \quad \text { and } \quad \hat{\psi}(x)=1-\sum_{j=1}^{\infty} \hat{\varphi}_{j}(x),
$$

where $\hat{f}$ denotes the Fourier transform of $f$, i.e., $\hat{f}(x):=\int_{\mathbb{R}^{n}} e^{-2 \pi i x \cdot \xi} f(\xi) d \xi$. Then for a positive measurable function $w$, the inhomogeneous Besov space $B_{p, w}^{s, q}\left(\mathbb{R}^{n}\right)$ and the homogeneous Besov space $\dot{B}_{p, w}^{s, q}\left(\mathbb{R}^{n}\right)$ are respectively defined by

$$
\left\{\begin{array}{l}
B_{p, w}^{s, q}\left(\mathbb{R}^{n}\right):=\left\{u \in \mathcal{S}^{\prime}\left(\mathbb{R}^{n}\right):\|u\|_{B_{p, w}^{s, q}}<\infty\right\}, \\
\|u\|_{B_{p, w}^{s, q}}:=\|\psi * u\|_{L_{p, w}}+\left(\sum_{j=1}^{\infty}\left(2^{s j}\left\|\varphi_{j} * u\right\|_{L_{p, w}}\right)^{q}\right)^{1 / q},
\end{array}\right.
$$


and

$$
\left\{\begin{array}{l}
\dot{B}_{p, w}^{s, q}\left(\mathbb{R}^{n}\right):=\left\{u \in\left(\mathcal{S}^{\prime} / \mathcal{P}\right)\left(\mathbb{R}^{n}\right):\|u\|_{\dot{B}_{p, w}^{s, q}}<\infty\right\} \\
\|u\|_{\dot{B}_{p, w}^{s, q}}:=\left(\sum_{j=-\infty}^{\infty}\left(2^{s j}\left\|\varphi_{j} * u\right\|_{L_{p, w}}\right)^{q}\right)^{1 / q}
\end{array}\right.
$$

for $s \in \mathbb{R}$ and $1 \leq p, q \leq \infty$. In the above definitions, $\mathcal{S}^{\prime}\left(\mathbb{R}^{n}\right)$ and $\mathcal{P}\left(\mathbb{R}^{n}\right)$ represent the classes of tempered distributions and of polynomials in $\mathbb{R}^{n}$, respectively. In addition, we make the usual modification if $q=\infty$, and $L_{p, w}\left(\mathbb{R}^{n}\right)$ denotes the weighted Lebesgue space as in 1.4 .

Next, we introduce inhomogeneous weighted Besov-Orlicz spaces, which are naturally defined by replacing weighted Lebesgue spaces with weighted Orlicz spaces in the definition 2.2 . Let $w$ be a positive measurable function, and let $\Phi$ be a Young function, that is, $\Phi$ is a continuous increasing convex function on $[0, \infty)$ satisfying $\Phi(0)=0$ and $\lim _{t \rightarrow \infty} \Phi(t)=\infty$. Then for $s \in \mathbb{R}$ and $1 \leq q \leq \infty$, the inhomogeneous weighted Besov-Orlicz space is defined by

$$
\left\{\begin{array}{l}
B_{\Phi, w}^{s, q}\left(\mathbb{R}^{n}\right):=\left\{u \in \mathcal{S}^{\prime}\left(\mathbb{R}^{n}\right):\|u\|_{B_{\Phi, w}^{s, q}}<\infty\right\} \\
\|u\|_{B_{\Phi, w}^{s, q}}:=\|\psi * u\|_{L_{\Phi, w}}+\left(\sum_{j=1}^{\infty}\left(2^{s j}\left\|\varphi_{j} * u\right\|_{L_{\Phi, w}}\right)^{q}\right)^{1 / q}
\end{array}\right.
$$

where we make the usual modification if $q=\infty$, and $L_{\Phi, w}\left(\mathbb{R}^{n}\right)$ denotes the weighted Orlicz space, which is a class of measurable functions defined by

$$
L_{\Phi, w}\left(\mathbb{R}^{n}\right):=\left\{f: \int_{\mathbb{R}^{n}} \Phi(\varepsilon|f|) w d x<\infty \text { for some } \varepsilon>0\right\}
$$

equipped with the Luxemburg norm

$$
\|f\|_{L_{\Phi, w}}:=\inf \left\{\lambda>0: \int_{\mathbb{R}^{n}} \Phi(|f| / \lambda) w d x \leq 1\right\} .
$$

Clearly, $B_{\Phi, w}^{s, q}\left(\mathbb{R}^{n}\right)$ coincides with $B_{p, w}^{s, q}\left(\mathbb{R}^{n}\right)$ for $\Phi(t)=t^{p}$ and $1 \leq p<\infty$.

In this section, we shall show two key lemmas, inspired by Rakotondratsimba [R1, R2], who proved weighted Young inequalities for convolutions with kernel functions behaving like the Riesz potential. However, for the purpose of the precise investigation of constants, we need to reconstruct this procedure with kernel functions belonging to the Schwartz class.

First, we recall the following $n$-dimensional Hardy type inequality:

Theorem A. (i) (n-dimensional Hardy type inequality) Let $U_{1}$ and $V_{1}$ be positive measurable weight functions in $\mathbb{R}^{n}, n \in \mathbb{N}$ and $1<p \leq q<\infty$. Then the inequality

$$
\left(\int_{\mathbb{R}^{n}}\left(\int_{\{2|y|<|x|\}} f(y) d y\right)^{q} U_{1}(x) d x\right)^{1 / q} \leq C_{1}\left(\int_{\mathbb{R}^{n}} f(x)^{p} V_{1}(x) d x\right)^{1 / p}
$$


holds for all $f \in L_{p, V_{1}}\left(\mathbb{R}^{n}\right)$ with $f \geq 0$ a.e. in $\mathbb{R}^{n}$ if and only if

$$
A_{1}:=\sup _{R>0}\left(\int_{\{|x|>2 R\}} U_{1}(x) d x\right)^{1 / q}\left(\int_{\{|x|<R\}} V_{1}(x)^{-\left(p^{\prime}-1\right)} d x\right)^{1 / p^{\prime}}<\infty .
$$

Moreover, the constant $C_{1}$ can be taken as

$$
C_{1}=\left(p^{\prime}\right)^{1 / p^{\prime}} p^{1 / q} A_{1} \text {. }
$$

(ii) (n-dimensional dual-Hardy type inequality) Let $U_{2}$ and $V_{2}$ be positive weight functions in $\mathbb{R}^{n}, n \in \mathbb{N}$ and $1<p \leq q<\infty$. Then the inequality

$$
\left(\int_{\mathbb{R}^{n}}\left(\int_{\{|y|>2|x|\}} f(y) d y\right)^{q} U_{2}(x) d x\right)^{1 / q} \leq C_{2}\left(\int_{\mathbb{R}^{n}} f(x)^{p} V_{2}(x) d x\right)^{1 / p}
$$

holds for all $f \in L_{p, V_{2}}\left(\mathbb{R}^{n}\right)$ with $f \geq 0$ a.e. in $\mathbb{R}^{n}$ if and only if

$$
A_{2}:=\sup _{R>0}\left(\int_{\{|x|<R\}} U_{2}(x) d x\right)^{1 / q}\left(\int_{\{|x|>2 R\}} V_{2}(x)^{-\left(p^{\prime}-1\right)} d x\right)^{1 / p^{\prime}}<\infty .
$$

Moreover, the constant $C_{2}$ can be taken as

$$
C_{2}=\left(p^{\prime}\right)^{1 / p^{\prime}} p^{1 / q} A_{2} \text {. }
$$

Indeed, Theorem $\mathrm{A}$ can be proved immediately from the $n$-dimensional Hardy and dual-Hardy inequalities shown by Drábek-Heinig-Kufner DHK, Theorem 2.1, p. 4] through scaling and changing variables.

We first prove a norm estimate with a subcritical homogeneous weight:

Lemma 2.1. (i) Let $\Psi \in \mathcal{S}\left(\mathbb{R}^{n}\right)$ be any fixed kernel function with $n \in \mathbb{N}$. Then

$$
\|\Psi * f\|_{L_{q, w_{r}}} \leq C_{n}\left(\frac{1}{n-r}\right)^{1 / q}\|f\|_{L_{p}} \quad \text { for all } f \in L_{p}\left(\mathbb{R}^{n}\right),
$$

$1<p \leq q<\infty$ and $0 \leq r<n$, where $w_{r}$ is the homogeneous weight as in (1.3).

(ii) Let $n \in \mathbb{N}$. Then

$$
\left\|\varphi_{j} * f\right\|_{L_{q, w_{r}}} \leq C_{n}\left(\frac{1}{n-r}\right)^{1 / q} 2^{\left(\frac{n}{p}-\frac{n-r}{q}\right) j}\|f\|_{L_{p}} \quad \text { for all } f \in L_{p}\left(\mathbb{R}^{n}\right),
$$

$1<p \leq q<\infty, 0 \leq r<n$ and $j \in \mathbb{Z}$, where $\left\{\varphi_{j}\right\}$ are the Schwartz functions as in 2.1.

Proof. Note that (ii) is an immediate consequence of (i). Indeed, since $\left(\varphi_{j} * f\right)\left(x / 2^{j}\right)=\left(\varphi_{0} *\left[f\left(\cdot / 2^{j}\right)\right]\right)(x)$, by applying (i) with $\Psi=\varphi_{0}$ we obtain

$$
\begin{aligned}
& \left\|\varphi_{j} * f\right\|_{L_{q, w_{r}}}=2^{-\frac{n-r}{q} j}\left\|\varphi_{0} *\left[f\left(\cdot / 2^{j}\right)\right]\right\|_{L_{q, w_{r}}} \\
& \quad \leq C_{n} 2^{-\frac{n-r}{q} j}\left(\frac{1}{n-r}\right)^{1 / q}\left\|f\left(\cdot / 2^{j}\right)\right\|_{L_{p}}=C_{n}\left(\frac{1}{n-r}\right)^{1 / q} 2^{\left(\frac{n}{p}-\frac{n-r}{q}\right) j}\|f\|_{L_{p}} .
\end{aligned}
$$


We turn to the proof of (i). Without loss of generality, we may assume $f \geq 0$ a.e. in $\mathbb{R}^{n}$, and that $\Psi$ is positive, since otherwise we can replace $\Psi$ by $|\Psi|+\eta$ with any fixed positive function $\eta \in \mathcal{S}\left(\mathbb{R}^{n}\right)$. Note that $\Psi$ might no longer be smooth after such a replacement. However, the only property we will need is its decay estimate, i.e., for any $\alpha \geq 0$, there exists a positive constant $C_{n, \alpha}$ such that

$$
0<|\Psi(x)|+\eta(x) \leq C_{n, \alpha}(1+|x|)^{-\alpha} \quad \text { for all } x \in \mathbb{R}^{n} .
$$

We first decompose the integral into three parts:

$$
\begin{aligned}
\|\Psi * f\|_{L_{q, w_{r}}}^{q} \leq & 3^{q}\left[\int_{\mathbb{R}^{n}}\left(\int_{\{|y|<|x| / 2\}} \Psi(x-y) f(y) d y\right)^{q} \frac{d x}{|x|^{r}}\right. \\
& +\int_{\mathbb{R}^{n}}\left(\int_{\{|x| / 2 \leq|y| \leq 2|x|\}} \Psi(x-y) f(y) d y\right)^{q} \frac{d x}{|x|^{r}} \\
& \left.+\int_{\mathbb{R}^{n}}\left(\int_{\{|y|>2|x|\}} \Psi(x-y) f(y) d y\right)^{q} \frac{d x}{|x|^{r}}\right] \\
=: & 3^{q}\left(I_{1}+I_{2}+I_{3}\right) .
\end{aligned}
$$

First, we estimate $I_{1}$. Note that $|y|<|x| / 2$ implies $|x| / 2<|x-y|$. Hence,

$$
I_{1} \leq \int_{\mathbb{R}^{n}}\left(\int_{\{|y|<|x| / 2\}} f(y) d y\right)^{q} \tilde{\Psi}(x)^{q} \frac{d x}{|x|^{r}} \text {, where } \tilde{\Psi}(x):=\sup _{\{|z|>|x| / 2\}} \Psi(z) .
$$

To apply Theorem A(i), we need to check the condition

$$
\left(\int_{\{2 R<|x|\}} \tilde{\Psi}(x)^{q} \frac{d x}{|x|^{r}}\right)^{1 / q}\left(\int_{\{|x|<R\}} d x\right)^{1 / p^{\prime}} \leq A_{1} \text { for all } R>0 .
$$

Indeed, once 2.9. has been verified, Theorem A(i) yields

$$
I_{1}^{1 / q} \leq\left(p^{\prime}\right)^{1 / p^{\prime}} p^{1 / q} A_{1}\|f\|_{L_{p}} \leq e^{2 / e} A_{1}\|f\|_{L_{p}} .
$$

Since $\Psi$ decays rapidly as $|x| \rightarrow \infty, \tilde{\Psi}$ also satisfies the same estimate as in (2.7), namely, for any $\alpha \geq 0$, there exists a positive constant $C_{n, \alpha}$ such that

$$
\tilde{\Psi}(x) \leq C_{n, \alpha}(1+|x|)^{-\alpha} \quad \text { for all } x \in \mathbb{R}^{n} .
$$

We now distinguish two cases:

CASE $1: R \geq 1$. Then by making use of the decay estimate 2.10 with $\alpha=2 n$, we see that 


$$
\begin{aligned}
\left(\int_{\{2 R<|x|\}} \tilde{\Psi}(x)^{q} \frac{d x}{|x|^{r}}\right)^{1 / q}\left(\int_{\{|x|<R\}} d x\right)^{1 / p^{\prime}} & \\
& \leq C_{n}\left(\int_{\{2 R<|x|\}}|x|^{-\alpha q-r} d x\right)^{1 / q}\left(\int_{\{|x|<R\}} d x\right)^{1 / p^{\prime}} \\
& \leq C_{n}\left(\frac{1}{\alpha q-(n-r)}\right)^{1 / q} R^{-\alpha+\frac{n-r}{q}+\frac{n}{p^{\prime}}} \leq C_{n},
\end{aligned}
$$

where we have used the facts that $R \geq 1$ and

$$
\left\{\begin{array}{l}
\alpha q-(n-r) \geq \alpha-(n-r)=n+r>0, \\
\left(\frac{1}{\alpha q-(n-r)}\right)^{1 / q} \leq\left(\frac{1}{n+r}\right)^{1 / q} \leq 1, \\
-\alpha+\frac{n-r}{q}+\frac{n}{p^{\prime}} \leq-2 n+(n-r)+n=-r \leq 0 .
\end{array}\right.
$$

Case 2: $0<R<1$. In this case,

$$
\begin{aligned}
\left(\int_{\{2 R<|x|\}} \tilde{\Psi}(x)^{q} \frac{d x}{|x|^{r}}\right)^{1 / q} & \\
& \leq\left(\int_{\{2 R<|x|<2\}} \tilde{\Psi}(x)^{q} \frac{d x}{|x|^{r}}\right)^{1 / q}+\left(\int_{\{|x| \geq 2\}} \tilde{\Psi}(x)^{q} \frac{d x}{|x|^{r}}\right)^{1 / q} \\
& =: J_{1}+J_{2} .
\end{aligned}
$$

First, by using 2.10 with $\alpha=0$, we obtain

$$
J_{1} \leq C_{n}\left(\int_{\{|x|<2\}} \frac{d x}{|x|^{r}}\right)^{1 / q} \leq C_{n}\left(\frac{1}{n-r}\right)^{1 / q} .
$$

Moreover, by 2.10 with $\alpha=n+1$,

$$
J_{2} \leq C_{n}\left(\int_{\{|x| \geq 2\}}|x|^{-\alpha q-r} d x\right)^{1 / q} \leq C_{n}\left(\frac{1}{\alpha q-(n-r)}\right)^{1 / q} \leq C_{n},
$$

where we have used the facts that

$$
\left\{\begin{array}{l}
\alpha q-(n-r) \geq \alpha-(n-r)=1+r>0 \\
\left(\frac{1}{\alpha q-(n-r)}\right)^{1 / q} \leq\left(\frac{1}{\alpha-(n-r)}\right)^{1 / q}=\left(\frac{1}{1+r}\right)^{1 / q} \leq 1 .
\end{array}\right.
$$

To sum up, we get 


$$
\begin{aligned}
\left(\int_{\{2 R<|x|\}} \tilde{\Psi}(x)^{q} \frac{d x}{|x|^{r}}\right)^{1 / q} & \left(\int_{\{|x|<R\}} d x\right)^{1 / p^{\prime}} \\
& \leq C_{n}\left(\frac{1}{n-r}\right)^{1 / q} R^{n / p^{\prime}} \leq C_{n}\left(\frac{1}{n-r}\right)^{1 / q}
\end{aligned}
$$

since $R<1$. Thus by (2.11), 2.12 and Theorem A(i), we obtain

$$
I_{1}^{1 / q} \leq C_{n}\left(\frac{1}{n-r}\right)^{1 / q}\|f\|_{L_{p}} .
$$

Next, we estimate $I_{3}$. Note that $2|x|<|y|$ implies $|y| / 2<|x-y|$. Thus

$$
I_{3} \leq \int_{\mathbb{R}^{n}}\left(\int_{\{|y|>2|x|\}} \tilde{\Psi}(y) f(y) d y\right)^{q} \frac{d x}{|x|^{r}} .
$$

To apply Theorem A(ii), we need to check the condition

$$
\left(\int_{\{|x|<R\}} \frac{d x}{|x|^{r}}\right)^{1 / q}\left(\int_{\{2 R<|x|\}}\left(\tilde{\Psi}(x)^{-p}\right)^{-\left(p^{\prime}-1\right)} d x\right)^{1 / p^{\prime}} \leq A_{2}
$$

for all $R>0$.

Indeed, once 2.14 has been verified, Theorem A(ii) yields

$$
I_{3}^{1 / q} \leq\left(p^{\prime}\right)^{1 / p^{\prime}} p^{1 / q} A_{2}\left(\int_{\mathbb{R}^{n}}(\tilde{\Psi}(x) f(x))^{p} \tilde{\Psi}(x)^{-p} d x\right)^{1 / p} \leq e^{2 / e} A_{2}\|f\|_{L_{p}} .
$$

Just as for $I_{1}$, we distinguish two cases:

CASE 1: $R \geq 1$. Then by 2.10 with $\alpha=2 n$,

$$
\begin{aligned}
\left(\int_{\{2 R<|x|\}} \tilde{\Psi}(x)^{p^{\prime}} d x\right)^{1 / p^{\prime}} & \leq C_{n}\left(\int_{\{2 R<|x|\}}|x|^{-\alpha p^{\prime}} d x\right)^{1 / p^{\prime}} \\
& \leq C_{n}\left(\frac{1}{\alpha p^{\prime}-n}\right)^{1 / p^{\prime}} R^{-\left(\alpha-n / p^{\prime}\right)} \leq C_{n} R^{-\left(\alpha-n / p^{\prime}\right)}
\end{aligned}
$$

where we have used the facts that

$$
\alpha p^{\prime}-n \geq \alpha-n=n>0, \quad\left(\frac{1}{\alpha p^{\prime}-n}\right)^{1 / p^{\prime}} \leq\left(\frac{1}{\alpha-n}\right)^{1 / p^{\prime}}=\left(\frac{1}{n}\right)^{1 / p^{\prime}} \leq 1 .
$$

Thus we obtain

$$
\begin{aligned}
\left(\int_{\{|x|<R\}} \frac{d x}{|x|^{r}}\right)^{1 / q} & \left(\int_{\{2 R<|x|\}} \tilde{\Psi}(x)^{p^{\prime}} d x\right)^{1 / p^{\prime}} \\
& \leq C_{n}\left(\frac{1}{n-r}\right)^{1 / q} R^{-\alpha+\frac{n-r}{q}+\frac{n}{p^{\prime}}} \leq C_{n}\left(\frac{1}{n-r}\right)^{1 / q}
\end{aligned}
$$

since $R \geq 1$ and $-\alpha+\frac{n-r}{q}+\frac{n}{p^{\prime}} \leq-2 n+(n-r)+n=-r \leq 0$. 
CASe 2: $0<R<1$. In this case,

$$
\begin{aligned}
\left(\int_{\{2 R<|x|\}} \tilde{\Psi}(x)^{p^{\prime}}\right. & d x)^{1 / p^{\prime}} \\
& \leq\left(\int_{\{2 R<|x|<2\}} \tilde{\Psi}(x)^{p^{\prime}} d x\right)^{1 / p^{\prime}}+\left(\int_{\{|x| \geq 2\}} \tilde{\Psi}(x)^{p^{\prime}} d x\right)^{1 / p^{\prime}} \\
& =: K_{1}+K_{2} .
\end{aligned}
$$

First, by 2.10 with $\alpha=0$, we obtain

$$
K_{1} \leq C_{n}\left(\int_{\{|x|<2\}} d x\right)^{1 / p^{\prime}} \leq C_{n} .
$$

Moreover, by $(2.10)$ with $\alpha=2 n$,

$$
K_{2} \leq C_{n}\left(\int_{\{|x| \geq 2\}}|x|^{-\alpha p^{\prime}} d x\right)^{1 / p^{\prime}} \leq C_{n}\left(\frac{1}{\alpha p^{\prime}-n}\right)^{1 / p^{\prime}} 2^{-\left(\alpha-n / p^{\prime}\right)} \leq C_{n} .
$$

To sum up, we get

$$
\begin{aligned}
& \left(\int_{\{|x|<R\}} \frac{d x}{|x|^{r}}\right)^{1 / q}\left(\int_{\{2 R<|x|\}} \tilde{\Psi}(x)^{p^{\prime}} d x\right)^{1 / p^{\prime}} \\
& \quad \leq C_{n}\left(\frac{1}{n-r}\right)^{1 / q} R^{(n-r) / q} \leq C_{n}\left(\frac{1}{n-r}\right)^{1 / q}
\end{aligned}
$$

since $R<1$. Thus by (2.15), 2.16) and Theorem A(ii), we obtain

$$
I_{3}^{1 / q} \leq C_{n}\left(\frac{1}{n-r}\right)^{1 / q}\|f\|_{L_{p}} .
$$

Finally, we estimate $I_{2}$. Since $|x| / 2 \leq|y| \leq 2|x|$ and $2^{k} \leq|x|<2^{k+1}$ imply $2^{k-1} \leq|y|<2^{k+2}$, we have

$$
\begin{aligned}
I_{2} & =\sum_{k \in \mathbb{Z}} \int_{\left\{2^{k} \leq|x|<2^{k+1}\right\}}\left(\int_{\{|x| / 2 \leq|y| \leq 2|x|\}} \Psi(x-y) f(y) d y\right)^{q} \frac{d x}{|x|^{r}} \\
& \leq \sum_{k \in \mathbb{Z}} 2^{-k r} \int_{\left\{2^{k} \leq|x|<2^{k+1}\right\}}\left(\Psi *\left[f \chi_{\left\{2^{k-1} \leq|\cdot|<2^{k+2}\right\}}\right]\right)(x)^{q} d x .
\end{aligned}
$$

Here, take $t:=n q /(n-r) \in[q, \infty)$. Then (2.18), Hölder's inequality and Young's inequality imply 


$$
\begin{aligned}
I_{2} \leq & C_{n} \sum_{k \in \mathbb{Z}} 2^{-k r+k n(1-q / t)} \\
& \times\left(\int_{\left\{2^{k} \leq|x|<2^{k+1}\right\}}\left(\Psi *\left[f \chi_{\left\{2^{k-1} \leq|\cdot|<2^{k+2}\right\}}\right]\right)(x)^{t} d x\right)^{q / t} \\
\leq & C_{n} \sum_{k \in \mathbb{Z}}\left\|\Psi *\left[f \chi_{\left\{2^{k-1} \leq|\cdot|<2^{k+2}\right\}}\right]\right\|_{L_{t}}^{q} \\
\leq & C_{n} \sum_{k \in \mathbb{Z}}\|\Psi\|_{L_{\tilde{t}}}^{q}\left\|f \chi_{\left\{2^{k-1} \leq|\cdot|<2^{k+2}\right\}}\right\|_{L_{p}}^{q} \\
\leq & C_{n}^{q} \sum_{k \in \mathbb{Z}}\left\|f \chi_{\left\{2^{k-1} \leq|\cdot|<2^{k+2}\right\}}\right\|_{L_{p}}^{q} \leq C_{n}^{q}\|f\|_{L_{p}}^{q},
\end{aligned}
$$

where $\tilde{t} \in\left[1, p^{\prime}\right)$ is the exponent satisfying $1 / t+1=1 / \tilde{t}+1 / p$. Moreover, the fact $\max _{1 \leq \tau \leq \infty}\|\Psi\|_{L_{\tau}}<\infty$ was used above. By (2.8), 2.13), 2.17) and (2.19), we obtain the desired estimate.

The following lemma yields a norm inequality involving the critical weight function $W_{s}$ defined in the statement of Theorem 1.2(ii):

Lemma 2.2. (i) Let $\Psi \in \mathcal{S}\left(\mathbb{R}^{n}\right)$ and $1<p, s<\infty$. Then

$$
\|\Psi * f\|_{L_{p, W_{s}, j}} \leq C_{n, p, s}\|f\|_{L_{p}} \quad \text { for all } f \in L_{p}\left(\mathbb{R}^{n}\right) \text { and } j \in \mathbb{N} \cup\{0\},
$$

where the weight function $W_{s, j}$ is defined by

$$
W_{s, j}(x):=\frac{1}{|x|^{n}\left[\log \left(e+2^{j} /|x|\right)\right]^{s}} \quad \text { for } x \in \mathbb{R}^{n} \backslash\{0\} .
$$

(ii) Let $n \in \mathbb{N}$ and $1<p, s<\infty$. Then

$\left\|\varphi_{j} * f\right\|_{L_{p, W_{s}}} \leq C_{n, p, s} 2^{\frac{n}{p} j}\|f\|_{L_{p}} \quad$ for all $f \in L_{p}\left(\mathbb{R}^{n}\right)$ and $j \in \mathbb{N} \cup\{0\}$, where $\left\{\varphi_{j}\right\}$ are the Schwartz functions as in (2.1), and the weight function $W_{s}$ is defined by

$$
W_{s}(x):=\frac{1}{|x|^{n}[\log (e+1 /|x|)]^{s}} \quad \text { for } x \in \mathbb{R}^{n} \backslash\{0\} .
$$

Proof. Note that (ii) is an immediate consequence of (i). Indeed, by noting $\left(\varphi_{j} * f\right)\left(x / 2^{j}\right)=\left(\varphi_{0} *\left[f\left(\cdot / 2^{j}\right)\right]\right)(x)$ and applying (i) with $\Psi=\varphi_{0}$, we see

$$
\begin{aligned}
\left\|\varphi_{j} * f\right\|_{L_{p, W_{s}}} & =\left\|\varphi_{0} *\left[f\left(\cdot / 2^{j}\right)\right]\right\|_{L_{p, W_{s, j}}} \leq C_{n, p, s}\left\|f\left(\cdot / 2^{j}\right)\right\|_{L_{p}} \\
& =C_{n, p, s} 2^{\frac{n}{p} j}\|f\|_{L_{p} .} .
\end{aligned}
$$

To prove (i), just as in the proof of Lemma 2.1, we may assume that $f \geq 0$ a.e. in $\mathbb{R}^{n}, \Psi$ is positive, and for any $\alpha \geq 0$, there exists $C_{n, \alpha}>0$ such that

$$
0<\Psi(x) \leq C_{n, \alpha}(1+|x|)^{-\alpha} \text { for all } x \in \mathbb{R}^{n} .
$$


Let us decompose the integral into three parts:

$$
\begin{aligned}
\|\Psi * f\|_{L_{p, W_{s}, j}}^{p} \leq & 3^{p}\left[\int_{\mathbb{R}^{n}}\left(\int_{\{|y|<|x| / 2\}} \Psi(x-y) f(y) d y\right)^{p} W_{s, j}(x) d x\right. \\
& +\int_{\mathbb{R}^{n}}\left(\int_{\{|x| / 2 \leq|y| \leq 2|x|\}} \Psi(x-y) f(y) d y\right)^{p} W_{s, j}(x) d x \\
& \left.+\int_{\mathbb{R}^{n}}\left(\int_{\{|y|>2|x|\}} \Psi(x-y) f(y) d y\right)^{p} W_{s, j}(x) d x\right] \\
=: & 3^{p}\left(L_{1}+L_{2}+L_{3}\right) .
\end{aligned}
$$

First, we estimate $L_{1}$. As $|y|<|x| / 2$ implies $|x| / 2<|x-y|$, we have $L_{1} \leq \int_{\mathbb{R}^{n}}\left(\int_{\{|y|<|x| / 2\}} f(y) d y\right)^{p} \tilde{\Psi}(x)^{p} W_{s, j}(x) d x$, where $\tilde{\Psi}(x):=\sup _{\{|z|>|x| / 2\}} \Psi(z)$.

To apply Theorem A(i), we need to check the condition

$$
\left(\int_{\{2 R<|x|\}} \tilde{\Psi}(x)^{p} W_{s, j}(x) d x\right)^{1 / p}\left(\int_{\{|x|<R\}} d x\right)^{1 / p^{\prime}} \leq A_{1} \quad \text { for all } R>0 .
$$

By 2.20 with any $\alpha>0$,

$$
\begin{aligned}
& \left(\int_{\{2 R<|x|\}} \tilde{\Psi}(x)^{p} W_{s, j}(x) d x\right)^{1 / p} \\
& \leq C_{n, \alpha}\left(\int_{\{2 R<|x|\}}|x|^{-\alpha p-n} \frac{d x}{\left[\log \left(e+2^{j} /|x|\right)\right]^{s}}\right)^{1 / p} \\
& \leq C_{n, \alpha}\left(\int_{\{2 R<|x|\}}|x|^{-\alpha p-n} d x\right)^{1 / p} \leq C_{n, \alpha} R^{-\alpha} \quad \text { for all } R>0 .
\end{aligned}
$$

Thus by taking $\alpha=n / p^{\prime}$ in 2.22 , we obtain

$$
\left(\int_{\{2 R<|x|\}} \tilde{\Psi}(x)^{p} W_{s, j}(x) d x\right)^{1 / p}\left(\int_{\{|x|<R\}} d x\right)^{1 / p^{\prime}} \leq C_{n, p} \quad \text { for all } R>0,
$$

and then Theorem A(i) yields

$$
L_{1}^{1 / p} \leq C_{n, p}\|f\|_{L_{p}} .
$$

Next, we estimate $L_{3}$. Since $2|x|<|y|$ implies $|y| / 2<|x-y|$, we have

$$
L_{3} \leq \int_{\mathbb{R}^{n}}\left(\int_{\{|y|>2|x|\}} \tilde{\Psi}(y) f(y) d y\right)^{p} W_{s, j}(x) d x .
$$


To apply Theorem A(ii), we need to check the condition $\left(\int_{\{|x|<R\}} W_{s, j}(x) d x\right)^{1 / p}\left(\int_{\{2 R<|x|\}}\left(\tilde{\Psi}(x)^{-p}\right)^{-\left(p^{\prime}-1\right)} d x\right)^{1 / p^{\prime}} \leq A_{2} \quad$ for all $R>0$. We distinguish two cases:

Case 1: $R>2^{j-1}$. In this case, $\int_{\{|x|<R\}} W_{s, j}(x) d x=\int_{\left\{|x|<2^{j-1}\right\}} W_{s, j}(x) d x+\int_{\left\{2^{j-1} \leq|x|<R\right\}} W_{s, j}(x) d x=: M_{1}+M_{2}$. By changing variables $x=2^{j} y$, it follows that

$$
\begin{aligned}
M_{1} & \leq \int_{\left\{|x|<2^{j-1}\right\}} \frac{d x}{|x|^{n}\left[\log \left(2^{j} /|x|\right)\right]^{s}}=\int_{\{|y|<1 / 2\}} \frac{d y}{|y|^{n}[\log (1 /|y|)]^{s}} \\
& =C_{n} \int_{\log 2}^{\infty} \frac{d \tau}{\tau^{s}}=C_{n, s},
\end{aligned}
$$

where the last integral in 2.24 is finite because of $s>1$. Next,

$$
M_{2} \leq \int_{\left\{2^{j-1} \leq|x|<R\right\}} \frac{d x}{|x|^{n}}=C_{n} \log \left(R / 2^{j-1}\right) .
$$

Moreover, by 2.20 with $\alpha=n / p^{\prime}+1$,

$$
\left(\int_{\{2 R<|x|\}} \tilde{\Psi}(x)^{p^{\prime}} d x\right)^{1 / p^{\prime}} \leq C_{n, \alpha}\left(\int_{\{2 R<|x|\}}|x|^{-\alpha p^{\prime}} d x\right)^{1 / p^{\prime}}=C_{n, p} R^{-1} .
$$

To sum up, we obtain

$$
\begin{aligned}
& \left(\int_{\{|x|<R\}} W_{s, j}(x) d x\right)^{1 / p}\left(\int_{\{2 R<|x|\}} \tilde{\Psi}(x)^{p^{\prime}} d x\right)^{1 / p^{\prime}} \\
& \quad \leq C_{n, p, s}\left[1+\log \left(R / 2^{j-1}\right)\right]^{1 / p} R^{-1} \\
& \quad=C_{n, p, s}\left[1+\log \left(R / 2^{j-1}\right)\right]^{1 / p}\left(R / 2^{j-1}\right)^{-1} 2^{-(j-1)} \leq C_{n, p, s}
\end{aligned}
$$

where we have used $j \in \mathbb{N} \cup\{0\}, R / 2^{j-1}>1$ and $\max _{1 \leq \tau<\infty}(1+\log \tau)^{1 / p} \tau^{-1}$ $<\infty$.

CASE 2: $R \leq 2^{j-1}$. In this case, by using (2.24) again, we have

$$
\left(\int_{\{|x|<R\}} W_{s, j}(x) d x\right)^{1 / p} \leq\left(\int_{\left\{|x|<2^{j-1}\right\}} W_{s, j}(x) d x\right)^{1 / p}=M_{1}^{1 / p} \leq C_{n, s} .
$$


Moreover, by 2.20 with $\alpha=n / p^{\prime}+1$,

$$
\begin{aligned}
\left(\int_{\{2 R<|x|\}} \tilde{\Psi}(x)^{p^{\prime}} d x\right)^{1 / p^{\prime}} & \leq C_{n, \alpha}\left(\int_{\{2 R<|x|\}}(1+|x|)^{-\alpha p^{\prime}} d x\right)^{1 / p^{\prime}} \\
& \leq C_{n, \alpha}\left(\int_{\mathbb{R}^{n}}(1+|x|)^{-\alpha p^{\prime}} d x\right)^{1 / p^{\prime}}=C_{n, p} .
\end{aligned}
$$

Thus we obtain

$$
\left(\int_{\{|x|<R\}} W_{s, j}(x) d x\right)^{1 / p}\left(\int_{\{2 R<|x|\}} \tilde{\Psi}(x)^{p^{\prime}} d x\right)^{1 / p^{\prime}} \leq C_{n, p, s} .
$$

Therefore, 2.25), 2.26) and Theorem A(ii) yield

$$
L_{3}^{1 / p} \leq C_{n, p, s}\|f\|_{L_{p}} .
$$

Finally, we estimate $L_{2}$. Since $|x| / 2 \leq|y| \leq 2|x|$ and $2^{k} \leq|x|<2^{k+1}$ imply $2^{k-1} \leq|y|<2^{k+2}$, by Young's inequality we have

$$
\begin{aligned}
L_{2} & =\sum_{k \in \mathbb{Z}} \int_{\left\{2^{k} \leq|x|<2^{k+1}\right\}}\left(\int_{\{|x| / 2 \leq|y| \leq 2|x|\}} \Psi(x-y) f(y) d y\right)^{p} W_{s, j}(x) d x \\
& \leq \sum_{k \in \mathbb{Z}} \int_{\left\{2^{k} \leq|x|<2^{k+1}\right\}}\left(\int_{\{|x| / 2 \leq|y| \leq 2|x|\}} \Psi(x-y) f(y) d y\right)^{p} \frac{d x}{|x|^{n}} \\
& \leq \sum_{k \in \mathbb{Z}} 2^{-k n} \int_{\left\{2^{k} \leq|x|<2^{k+1}\right\}}\left(\Psi *\left[f \chi_{\left\{2^{k-1} \leq|\cdot|<2^{k+2}\right\}}\right]\right)(x)^{p} d x \\
& \leq C_{n} \sum_{k \in \mathbb{Z}}\left\|\Psi *\left[f \chi_{\left\{2^{k-1} \leq|\cdot|<2^{k+2}\right\}}\right]\right\|_{L_{\infty}}^{p} \\
& \leq C_{n} \sum_{k \in \mathbb{Z}}\|\Psi\|_{L_{p^{\prime}}}^{p}\left\|f \chi_{\left\{2^{k-1} \leq|\cdot|<2^{k+2}\right\}}\right\|_{L_{p}}^{p} \\
& \leq C_{n}^{p} \sum_{k \in \mathbb{Z}}\left\|f \chi_{\left\{2^{k-1} \leq|\cdot|<2^{k+2}\right\}}\right\|_{L_{p}}^{p} \leq C_{n}^{p}\|f\|_{L_{p}}^{p},
\end{aligned}
$$

where we have used $\max _{1 \leq \tau \leq \infty}\|\Psi\|_{L_{\tau}}<\infty$. Hence, by (2.21), 2.23), 2.27) and (2.28), we obtain the desired estimate.

\section{Proofs of theorems}

Proof of Theorem 1.1. We consider only the case $\nu<\min \left\{\rho_{1}, \rho_{2}\right\} \leq$ $\max \left\{\rho_{1}, \rho_{2}\right\}<\infty$ since the limiting cases can be obtained quite similarly.

By using the partition of unity $\sum_{j \in \mathbb{Z}} \hat{\varphi}_{j} \equiv 1$, we decompose

$$
u=\sum_{j=-\infty}^{Z-1} \varphi_{j} * u+\sum_{j=Z}^{\infty} \varphi_{j} * u=: u_{1}+u_{2} \quad \text { for any fixed } Z \in \mathbb{Z} .
$$


We first estimate $\left\|u_{1}\right\|_{\dot{B}_{q, w_{r}}^{0, \nu}}$. Since $\operatorname{supp} \hat{\varphi}_{j}=\left\{2^{j-1} \leq|x| \leq 2^{j+1}\right\}$, we see that $\varphi_{j} * \varphi_{l} \equiv 0$ if $|j-l|>1$. Therefore,

$$
\begin{aligned}
\left\|u_{1}\right\|_{\dot{B}_{q, w_{r}}^{0, \nu}} & =\left(\sum_{l=-\infty}^{\infty}\left\|\varphi_{l} * u_{1}\right\|_{L_{q, w_{r}}}^{\nu}\right)^{1 / \nu}=\left(\sum_{l=-\infty}^{Z}\left\|\varphi_{l} * u_{1}\right\|_{L_{q, w_{r}}}^{\nu}\right)^{1 / \nu} \\
& =\left(\sum_{l=-\infty}^{Z}\left\|\tilde{\varphi}_{l} * \varphi_{l} * u_{1}\right\|_{L_{q, w_{r}}}^{\nu}\right)^{1 / \nu}
\end{aligned}
$$

where $\tilde{\varphi}_{l}:=\varphi_{l-1}+\varphi_{l}+\varphi_{l+1}$. By Lemma 2.1(ii) and Hölder's inequality,

$$
\begin{aligned}
& \left\|u_{1}\right\|_{\dot{B}_{q, w_{r}}^{0, \nu}} \leq C_{n}\left(\frac{1}{n-r}\right)^{1 / q}\left(\sum_{l=-\infty}^{Z} 2^{\left(\frac{n}{p}-\frac{n-r}{q}\right) l \nu}\left\|\varphi_{l} * u_{1}\right\|_{L_{p}}^{\nu}\right)^{1 / \nu} \\
& \leq C_{n}\left(\frac{1}{n-r}\right)^{1 / q}\left(\sum_{l=-\infty}^{Z} 2^{\left(\frac{n}{p}-\frac{n-r}{q}\right) \cdot \frac{\rho_{1} \nu}{\rho_{1}-\nu} l}\right)^{\frac{\rho_{1}-\nu}{\rho_{1} \nu}}\left(\sum_{l=-\infty}^{Z}\left\|\varphi_{l} * u_{1}\right\|_{L_{p}}^{\rho_{1}}\right)^{1 / \rho_{1}} \\
& \leq C_{n}\left(\frac{1}{n-r}\right)^{1 / q}\left(\sum_{l=-\infty}^{Z} 2^{\left(\frac{n}{p}-\frac{n-r}{q}\right) \cdot \frac{\rho_{1} \nu}{\rho_{1}-\nu} l}\right)^{\frac{\rho_{1}-\nu}{\rho_{1} \nu}}\left\|u_{1}\right\|_{\dot{B}_{p}^{0, \rho_{1}}} .
\end{aligned}
$$

Moreover, by Young's inequality,

$$
\begin{aligned}
\left\|u_{1}\right\|_{\dot{B}_{p}^{0, \rho_{1}}} & =\left(\sum_{l=-\infty}^{\infty}\left\|\varphi_{l} *\left(\sum_{j=-\infty}^{Z-1} \varphi_{j} * u\right)\right\|_{L_{p}}^{\rho_{1}}\right)^{1 / \rho_{1}} \\
& \leq\left(\sum_{l=-\infty}^{\infty}\left(\sum_{j=l-1}^{l+1}\left\|\varphi_{l} * \varphi_{j} * u\right\|_{L_{p}}\right)^{\rho_{1}}\right)^{1 / \rho_{1}} \\
& \leq\left(\sum_{l=-\infty}^{\infty}\left(\sum_{j=l-1}^{l+1}\left\|\varphi_{j}\right\|_{L_{1}}\left\|\varphi_{l} * u\right\|_{L_{p}}\right)^{\rho_{1}}\right)^{1 / \rho_{1}} \\
& =3\left\|\varphi_{0}\right\|_{L_{1}}\left(\sum_{l=-\infty}^{\infty}\left\|\varphi_{l} * u\right\|_{L_{p}}^{\rho_{1}}\right)^{1 / \rho_{1}}=3\left\|\varphi_{0}\right\|_{L_{1}}\|u\|_{\dot{B}_{p}^{0, \rho_{1}}},
\end{aligned}
$$

where we have used the fact that $\left\|\varphi_{j}\right\|_{L_{1}}=\left\|\varphi_{0}\right\|_{L_{1}}$ for all $j \in \mathbb{Z}$. Next, we investigate the geometric series which appears in (3.1). Since $t /\left(2^{t}-1\right) \leq$ $1 / \log 2$ for all $t>0$, we see that

$$
\begin{aligned}
\left(\sum_{l=-\infty}^{Z} 2^{\left(\frac{n}{p}-\frac{n-r}{q}\right) \cdot \frac{\rho_{1} \nu}{\rho_{1}-\nu} l}\right)^{\frac{\rho_{1}-\nu}{\rho_{1} \nu}} & \\
& =2^{\left(\frac{n}{p}-\frac{n-r}{q}\right)(Z+1)}\left(\frac{1}{2^{\left(\frac{n}{p}-\frac{n-r}{q}\right) \cdot \frac{\rho_{1} \nu}{\rho_{1}-\nu}}-1}\right)^{\frac{\rho_{1}-\nu}{\rho_{1} \nu}}
\end{aligned}
$$




$$
\begin{aligned}
& \leq C_{n} 2^{\left(\frac{n}{p}-\frac{n-r}{q}\right) Z}\left(\frac{1}{\left(\frac{n}{p}-\frac{n-r}{q}\right) \cdot \frac{\rho_{1} \nu}{\rho_{1}-\nu}}\right)^{\frac{\rho_{1}-\nu}{\rho_{1} \nu}} \\
& \leq C_{n} 2^{\left(\frac{n}{p}-\frac{n-r}{q}\right) Z}\left(\frac{p \cdot \frac{q}{p}}{n \cdot \frac{q}{p}-(n-r)}\right)^{\frac{\rho_{1}-\nu}{\rho_{1} \nu}} \\
& \leq C_{n} 2^{\left(\frac{n}{p}-\frac{n-r}{q}\right) Z} p^{\frac{\rho_{1}-\nu}{\rho_{1} \nu}}\left[\sup _{t \geq 1} \frac{t}{n t-(n-r)}\right]^{\frac{\rho_{1}-\nu}{\rho_{1} \nu}} \\
& \leq C_{n} 2^{\left(\frac{n}{p}-\frac{n-r}{q}\right) Z}\left(\frac{q}{r}\right)^{\frac{\rho_{1}-\nu}{\rho_{1} \nu}}
\end{aligned}
$$

where we have used the fact that $\sup _{t \geq 1} t /(n t-(n-r))=1 / r$. Summing up, by (3.1)-(3.3), we get

$$
\left\|u_{1}\right\|_{\dot{B}_{q, w_{r}}^{0, \nu}} \leq C_{n}\left(\frac{1}{n-r}\right)^{1 / q}\left(\frac{q}{r}\right)^{\frac{1}{\nu}-\frac{1}{\rho_{1}}} 2^{\left(\frac{n}{p}-\frac{n-r}{q}\right) Z}\|u\|_{\dot{B}_{p}^{0, \rho_{1}}}
$$

for all $Z \in \mathbb{Z}$.

Next,

$$
\begin{aligned}
\left\|u_{2}\right\|_{\dot{B}_{q, w_{r}}^{0, \nu}} & =\left(\sum_{l=-\infty}^{\infty}\left\|\varphi_{l} * u_{2}\right\|_{L_{q, w_{r}}}^{\nu}\right)^{1 / \nu}=\left(\sum_{l=Z-1}^{\infty}\left\|\varphi_{l} * u_{2}\right\|_{L_{q, w_{r}}}^{\nu}\right)^{1 / \nu} \\
& =\left(\sum_{l=Z-1}^{\infty}\left\|\tilde{\varphi}_{l} * \varphi_{l} * u_{2}\right\|_{L_{q, w_{r}}}^{\nu}\right)^{1 / \nu} \cdot
\end{aligned}
$$

By Lemma 2.1(ii) and Hölder's inequality,

$$
\begin{aligned}
\left\|u_{2}\right\|_{\dot{B}_{q, w_{r}}^{0, \nu} \leq} \leq & C_{n}\left(\frac{1}{n-r}\right)^{1 / q}\left(\sum_{l=Z-1}^{\infty} 2^{\left(\frac{n}{p}-\frac{n-r}{q}\right) l \nu}\left\|\varphi_{l} * u_{2}\right\|_{L_{p}}^{\nu}\right)^{1 / \nu} \\
= & C_{n}\left(\frac{1}{n-r}\right)^{1 / q}\left(\sum_{l=Z-1}^{\infty} 2^{-\frac{n-r}{q} l \nu}\left(2^{\frac{n}{p} l}\left\|\varphi_{k} * u_{2}\right\|_{L_{p}}\right)^{\nu}\right)^{1 / \nu} \\
\leq & C_{n}\left(\frac{1}{n-r}\right)^{1 / q}\left(\sum_{l=Z-1}^{\infty} 2^{-\frac{n-r}{q} \cdot \frac{\rho_{2} \nu}{\rho_{2}-\nu} l}\right)^{\frac{\rho_{2}-\nu}{\rho_{2} \nu}} \\
& \times\left(\sum_{l=Z-1}^{\infty}\left(2^{\frac{n}{p} l}\left\|\varphi_{l} * u_{2}\right\|_{\left.L_{p}\right)^{\rho_{2}}}\right)^{1 / \rho_{2}}\right. \\
\leq & C_{n}\left(\frac{1}{n-r}\right)^{1 / q}\left(\sum_{l=Z-1}^{\infty} 2^{-\frac{n-r}{q} \cdot \frac{\rho_{2} \nu}{\rho_{2}-\nu} l}\right)^{\frac{\rho_{2}-\nu}{\rho_{2} \nu}}\left\|u_{2}\right\|_{\dot{B}_{p}^{n / p, \rho_{2}}}
\end{aligned}
$$

Quite similarly as in $\left(3.2\right.$, we obtain $\left\|u_{2}\right\|_{\dot{B}_{p}^{n / p, \rho_{2}}} \leq 3\left\|\varphi_{0}\right\|_{L_{1}}\|u\|_{\dot{B}_{p}^{n / p, \rho_{2}}}$. Since 
$t /\left(2^{t}-1\right) \leq 1 / \log 2$ for all $t>0$, the geometric series in $(3.5)$ is estimated as follows:

$$
\begin{aligned}
& \left(\sum_{l=Z-1}^{\infty} 2^{-\frac{n-r}{q} \cdot \frac{\rho_{2} \nu}{\rho_{2}-\nu} l}\right)^{\frac{\rho_{2}-\nu}{\rho_{2} \nu}} \\
& \quad=2^{-\frac{n-r}{q}(Z-1)}\left(\frac{1}{1-2^{-\frac{n-r}{q} \cdot \frac{\rho_{2} \nu}{\rho_{2}-\nu}}}\right)^{\frac{\rho_{2}-\nu}{\rho_{2} \nu}} \leq C_{n} 2^{-\frac{n-r}{q} Z}\left(\frac{1}{2^{\frac{n-r}{q} \cdot \frac{\rho_{2} \nu}{\rho_{2}-\nu}}-1}\right)^{\frac{\rho_{2}-\nu}{\rho_{2} \nu}} \\
& \quad \leq C_{n} 2^{-\frac{n-r}{q} Z}\left(\frac{q}{n-r} \cdot \frac{\rho_{2}-\nu}{\rho_{2} \nu}\right)^{\frac{\rho_{2}-\nu}{\rho_{2} \nu}} \leq C_{n} 2^{-\frac{n-r}{q} Z}\left(\frac{q}{n-r}\right)^{\frac{\rho_{2}-\nu}{\rho_{2} \nu}} .
\end{aligned}
$$

To sum up, we get

$$
\left\|u_{2}\right\|_{\dot{B}_{q, w_{r}}^{0, \nu}} \leq C_{n} 2^{-\frac{n-r}{q} Z}\left(\frac{1}{n-r}\right)^{\frac{1}{q}+\frac{1}{\nu}-\frac{1}{\rho_{2}}} q^{\frac{1}{\nu}-\frac{1}{\rho_{2}}}\|u\|_{\dot{B}_{p}^{n / p, \rho_{2}}}
$$

for all $Z \in \mathbb{Z}$.

Combining (3.4) with (3.6), we have, for any $Z \in \mathbb{Z}$,

$$
\begin{aligned}
\|u\|_{\dot{B}_{q, w_{r}}^{0, \nu} \leq} \leq & C_{n}\left(\frac{1}{r}\right)^{\frac{1}{\nu}-\frac{1}{\rho_{1}}}\left(\frac{1}{n-r}\right)^{\frac{1}{q}+\frac{1}{\nu}-\frac{1}{\rho_{2}}} q^{\frac{1}{\nu}-\frac{1}{\max \left\{\rho_{1}, \rho_{2}\right\}}} \\
& \times\left(2^{\left(\frac{n}{p}-\frac{n-r}{q}\right) Z}\|u\|_{\dot{B}_{p}^{0, \rho_{1}}}+2^{-\frac{n-r}{q} Z}\|u\|_{\dot{B}_{p}^{n / p, \rho_{2}}}\right) .
\end{aligned}
$$

Furthermore, as $[t] \leq t<[t]+1$ for all $t \in \mathbb{R}$ where $[t]$ denotes the integer part, it follows that

$$
\begin{aligned}
\|u\|_{\dot{B}_{q, w_{r}}^{0, \nu}} \leq & C_{n}\left(\frac{1}{r}\right)^{\frac{1}{\nu}-\frac{1}{\rho_{1}}}\left(\frac{1}{n-r}\right)^{\frac{1}{q}+\frac{1}{\nu}-\frac{1}{\rho_{2}}} q^{\frac{1}{\nu}-\frac{1}{\max \left\{\rho_{1}, \rho_{2}\right\}}} \\
& \times\left(2^{\left(\frac{n}{p}-\frac{n-r}{q}\right) t}\|u\|_{\dot{B}_{p}^{0, \rho_{1}}}+2^{-\frac{n-r}{q} t}\|u\|_{\dot{B}_{p}^{n / p, \rho_{2}}}\right)
\end{aligned}
$$

for all $t \in \mathbb{R}$. In the end, we take $t=t_{0}$ in $(3.8)$ with

$$
t_{0}:=\frac{p}{n \log 2} \log \left(\frac{\|u\|_{\dot{B}_{p}^{n / p, \rho_{2}}}}{\|u\|_{\dot{B}_{p}^{0, \rho_{1}}}}\right)
$$

to obtain

$$
2^{\left(\frac{n}{p}-\frac{n-r}{q}\right) t_{0}}\|u\|_{\dot{B}_{p}^{0, \rho_{1}}}=2^{-\frac{n-r}{q} t_{0}}\|u\|_{\dot{B}_{p}^{n / p, \rho_{2}}}=\|u\|_{\dot{B}_{p}^{0, \rho_{1}}}^{\frac{(n-r) p}{n q}}\|u\|_{\dot{B}_{p}^{n / p, \rho_{2}}}^{1-\frac{(n-r) p}{n q}},
$$

which finishes the proof.

Proof of Theorem 1.2. We deal only with the case $\rho<\infty$ since the limiting case $\rho=\infty$ requires no modification. First, we prove (i). By the 
definition of the inhomogeneous weighted Besov space, we have

$$
\|u\|_{B_{p, w_{r}}^{0, \rho}}=\|\psi * u\|_{L_{p, w_{r}}}+\left(\sum_{j=1}^{\infty}\left\|\varphi_{j} * u\right\|_{L_{p, w_{r}}}^{\rho}\right)^{1 / \rho} .
$$

By noting $\psi=\sum_{j=-\infty}^{0} \varphi_{j}$, and applying Lemma 2.1(i) with $p=q$ and $\Psi=\sum_{l=-\infty}^{1} \varphi_{l}=\psi+\varphi_{1}$, we see that

$$
\begin{aligned}
\|\psi * u\|_{L_{p, w_{r}}} & =\left\|\left(\sum_{l=-\infty}^{1} \varphi_{l}\right) * \psi * u\right\|_{L_{p, w_{r}}} \\
& \leq C_{n}\left(\frac{1}{n-r}\right)^{1 / p}\|\psi * u\|_{L_{p}} .
\end{aligned}
$$

Furthermore, by making use of Lemma 2.1(ii) with $p=q$, we obtain

$$
\begin{aligned}
\left(\sum_{j=1}^{\infty}\left\|\varphi_{j} * u\right\|_{L_{p, w_{r}}}^{\rho}\right)^{1 / \rho} & =\left(\sum_{j=1}^{\infty}\left\|\tilde{\varphi}_{j} * \varphi_{j} * u\right\|_{L_{p, w_{r}}}^{\rho}\right)^{1 / \rho} \\
& \leq C_{n}\left(\frac{1}{n-r}\right)^{1 / p}\left(\sum_{j=1}^{\infty}\left(2^{\frac{r}{p} j}\left\|\varphi_{j} * u\right\|_{L_{p}}\right)^{\rho}\right)^{1 / \rho}
\end{aligned}
$$

Hence, combining (3.9) with $(3.10)$, we get the desired estimate.

Next, we prove (ii). First, by Lemma 2.2 (i) with $j=0$ and $\Psi=\sum_{l=-\infty}^{1} \varphi_{l}$,

$$
\|\psi * u\|_{L_{p, W_{s}}}=\left\|\left(\sum_{l=-\infty}^{1} \varphi_{l}\right) * \psi * u\right\|_{L_{p, W_{s}}} \leq C_{n, p, s}\|\psi * u\|_{L_{p}} .
$$

Furthermore, by Lemma 2.2 (ii),

$$
\begin{aligned}
\left(\sum_{j=1}^{\infty}\left\|\varphi_{j} * u\right\|_{L_{p, W_{s}}}^{\rho}\right)^{1 / \rho} & =\left(\sum_{j=1}^{\infty}\left\|\tilde{\varphi}_{j} * \varphi_{j} * u\right\|_{L_{p, W_{s}}}^{\rho}\right)^{1 / \rho} \\
& \leq C_{n, p, s}\left(\sum_{j=1}^{\infty}\left(2^{\frac{n}{p} j}\left\|\varphi_{j} * u\right\|_{L_{p}}\right)^{\rho}\right)^{1 / \rho} .
\end{aligned}
$$

Hence, combining (3.11) with (3.12), we get the desired estimate.

Proof of Theorem 1.3. We deal only with the case $\rho<\infty$ since the limiting case $\rho=\infty$ is similar. We first compute the weighted Orlicz norm $\|\psi * u\|_{L_{\Phi_{0}, w_{r}}}$. Let $\varepsilon>0$, to be determined later. By Lemma 2.1(i) with $\Psi=\sum_{l=-\infty}^{1} \varphi_{l}=\psi+\varphi_{1}$, 


$$
\begin{aligned}
\int_{\mathbb{R}^{n}} \Phi_{0}(\varepsilon|\psi * u|) w_{r} d x & =\sum_{k=k_{0}}^{\infty} \frac{\varepsilon^{(\mu-\delta) k}}{k !}\|\psi * u\|_{L_{(\mu-\delta) k, w_{r}}^{(\mu-\delta) k}}^{\infty} \\
& =\sum_{k=k_{0}}^{\infty} \frac{\varepsilon^{(\mu-\delta) k}}{k !}\left\|\left(\sum_{l=-\infty}^{1} \varphi_{l}\right) * \psi * u\right\|_{L_{(\mu-\delta) k, w_{r}}}^{(\mu-\delta) k} \\
& \leq \frac{1}{n-r} \sum_{k=k_{0}}^{\infty} \frac{\left(\varepsilon C_{n}\|\psi * u\|_{L_{p}}\right)^{(\mu-\delta) k}}{k !} \\
& \leq \frac{1}{n-r} \sum_{k=1}^{\infty} \frac{\left(\varepsilon C_{n}\|\psi * u\|_{L_{p}}\right)^{(\mu-\delta) k}}{k !} \\
& =\frac{1}{n-r}\left[\exp \left(\left(\varepsilon C_{n}\|\psi * u\|_{L_{p}}\right)^{\mu-\delta}\right)-1\right] .
\end{aligned}
$$

We now take $\varepsilon=\varepsilon_{0}>0$ satisfying

$$
\frac{1}{n-r}\left[\exp \left(\left(\varepsilon_{0} C_{n}\|\psi * u\|_{L_{p}}\right)^{\mu-\delta}\right)-1\right]=1,
$$

so that

$$
\varepsilon_{0}=\frac{[\log (1+n-r)]^{1 /(\mu-\delta)}}{C_{n}\|\psi * u\|_{L_{p}}} .
$$

Thus by the definition of the Luxemburg norm, we have

$$
\|\psi * u\|_{L_{\Phi_{0}, w_{r}}} \leq \frac{1}{\varepsilon_{0}}=\frac{C_{n}\|\psi * u\|_{L_{p}}}{[\log (1+n-r)]^{1 /(\mu-\delta)}} .
$$

Here, we use the following elementary inequality: for any $a>0$,

$$
\frac{t}{\log (1+t)} \leq \frac{a}{\log (1+a)} \quad \text { for all } 0<t \leq a \text {. }
$$

Hence, by using (3.13), (3.14) and $\mu-\delta \geq 1$, we obtain

$$
\|\psi * u\|_{L_{\Phi_{0}, w_{r}}} \leq C_{n}\left(\frac{1}{n-r}\right)^{1 /(\mu-\delta)}\|\psi * u\|_{L_{p}} .
$$

Next, we handle $\left\|\varphi_{j} * u\right\|_{L_{\Phi_{0}, w_{r}}}$ for each fixed $j \in \mathbb{Z}$ in the same way as when estimating of $\|\psi * u\|_{L_{\Phi_{0}, w_{r}}}$. Let $\varepsilon>0$, to be chosen later. Then by Lemma 2.1(ii),

$$
\begin{aligned}
\int_{\mathbb{R}^{n}} \Phi_{0}\left(\varepsilon\left|\varphi_{j} * u\right|\right) w_{r} d x & =\sum_{k=k_{0}}^{\infty} \frac{\varepsilon^{(\mu-\delta) k}}{k !}\left\|\varphi_{j} * u\right\|_{L_{(\mu-\delta) k, w_{r}}}^{(\mu-\delta) k} \\
& =\sum_{k=k_{0}}^{\infty} \frac{\varepsilon^{(\mu-\delta) k}}{k !}\left\|\tilde{\varphi}_{j} * \varphi_{j} * u\right\|_{L_{(\mu-\delta) k, w_{r}}}^{(\mu-\delta) k}
\end{aligned}
$$




$$
\begin{aligned}
& \leq \frac{1}{(n-r) 2^{(n-r) j}} \sum_{k=k_{0}}^{\infty} \frac{\left(\varepsilon C_{n} 2^{\frac{n}{p} j}\left\|\varphi_{j} * u\right\|_{L_{p}}\right)^{(\mu-\delta) k}}{k !} \\
& \leq \frac{1}{(n-r) 2^{(n-r) j}} \sum_{k=1}^{\infty} \frac{\left(\varepsilon C_{n} 2^{\frac{n}{p} j}\left\|\varphi_{j} * u\right\|_{L_{p}}\right)^{(\mu-\delta) k}}{k !} \\
& =\frac{1}{(n-r) 2^{(n-r) j}}\left[\exp \left(\left(\varepsilon C_{n} 2^{\frac{n}{p} j}\left\|\varphi_{j} * u\right\|_{L_{p}}\right)^{\mu-\delta}\right)-1\right] .
\end{aligned}
$$

In particular, we take $\varepsilon=\varepsilon_{0}>0$ satisfying

$$
\frac{1}{(n-r) 2^{(n-r) j}}\left[\exp \left(\left(\varepsilon_{0} C_{n} 2^{\frac{n}{p} j}\left\|\varphi_{j} * u\right\|_{L_{p}}\right)^{\mu-\delta}\right)-1\right]=1,
$$

so that

$$
\varepsilon_{0}=\frac{\left[\log \left(1+(n-r) 2^{(n-r) j}\right)\right]^{1 /(\mu-\delta)}}{C_{n} 2^{\frac{n}{p} j}\left\|\varphi_{j} * u\right\|_{L_{p}}} .
$$

Thus by the definition of the Luxemburg norm, we have

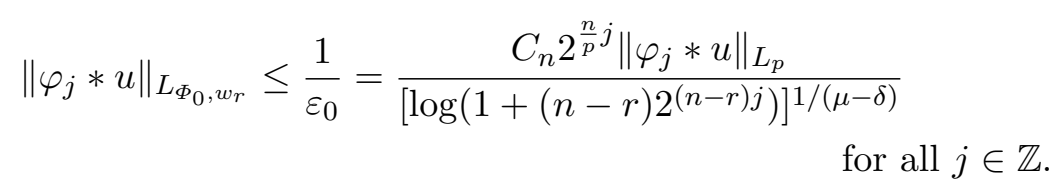

Therefore, by (3.16) and Hölder's inequality,

$$
\begin{aligned}
& \left(\sum_{j=1}^{\infty}\left\|\varphi_{j} * u\right\|_{L_{\Phi_{0}, w_{r}}}^{\nu}\right)^{1 / \nu} \\
\leq & C_{n}\left[\sum_{j=1}^{\infty}\left[\log \left(1+(n-r) 2^{(n-r) j}\right)\right]^{-\frac{\nu}{\mu-\delta}}\left(2^{\frac{n}{p} j}\left\|\varphi_{j} * u\right\|_{L_{p}}\right)^{\nu}\right]^{1 / \nu} \\
\leq & C_{n}\left[\sum_{j=1}^{\infty}\left[\log \left(1+(n-r) 2^{(n-r) j}\right)\right]^{-\frac{\mu}{\mu-\delta}}\right]^{1 / \mu}\left[\sum_{j=1}^{\infty}\left(2^{\frac{n}{p} j}\left\|\varphi_{j} * u\right\|_{L_{p}}\right)^{\rho}\right]^{1 / \rho} .
\end{aligned}
$$

In what follows, we investigate the non-negative term series on the righthand side of (3.17). For a technical reason, take $t_{0} \in \mathbb{R}$ defined by the equation

$$
\log (n-r)+t_{0}(n-r) \log 2=\frac{t_{0}(n-r) \log 2}{1+\mu / \delta},
$$

so that

$$
t_{0}=\frac{\mu+\delta}{\mu(\log 2)(n-r)} \log \left(\frac{1}{n-r}\right) .
$$

We distinguish two cases:

CASE 1: $t_{0} \geq 1$. Noting that $\mu>1$, we decompose the sum into two parts: 


$$
\begin{aligned}
{\left[\sum_{j=1}^{\infty}[\log (1\right.} & \left.\left.\left.+(n-r) 2^{(n-r) j}\right)\right]^{-\frac{\mu}{\mu-\delta}}\right]^{1 / \mu} \\
\leq & {\left[\sum_{j=1}^{\left[t_{0}\right]}\left[\log \left(1+(n-r) 2^{(n-r) j}\right)\right]^{-\frac{\mu}{\mu-\delta}}\right]^{1 / \mu} } \\
& +\left[\sum_{j=\left[t_{0}\right]+1}^{\infty}\left[\log \left(1+(n-r) 2^{(n-r) j}\right)\right]^{-\frac{\mu}{\mu-\delta}}\right]^{1 / \mu}=: N_{1}+N_{2} .
\end{aligned}
$$

We first estimate $N_{1}$. By (3.14), we have

$$
\begin{aligned}
\frac{(n-r) 2^{(n-r) j}}{\log \left(1+(n-r) 2^{(n-r) j}\right)} \leq \frac{(n-r) 2^{(n-r)\left[t_{0}\right]}}{\log \left(1+(n-r) 2^{(n-r)\left[t_{0}\right]}\right)} & \text { for all } 1 \leq j \leq\left[t_{0}\right] .
\end{aligned}
$$

Moreover, direct computation yields

$$
\begin{aligned}
& (n-r) 2^{(n-r)\left[t_{0}\right]} \leq(n-r) 2^{(n-r) t_{0}}=\left(\frac{1}{n-r}\right)^{\delta / \mu}, \\
& \frac{1}{\log \left(1+(n-r) 2^{(n-r)\left[t_{0}\right]}\right)} \leq \frac{1}{\log \left(1+(n-r) 2^{(n-r)\left(t_{0}-1\right)}\right)} \\
& =\frac{1}{\log \left(1+2^{-(n-r)}(n-r)^{-\delta / \mu}\right)} \leq 1 / \log \left(1+2^{-n} n^{-1}\right),
\end{aligned}
$$

where we have used the facts that $\left[t_{0}\right] \leq t_{0}<\left[t_{0}\right]+1$ and $\delta / \mu \leq 1-1 / \mu<1$. Hence, by (3.18)-3.20),

$$
\begin{aligned}
& \log \left(1+(n-r) 2^{(n-r) j}\right) \geq C_{n}(n-r)^{(\mu+\delta) / \mu} 2^{(n-r) j} \\
& \qquad \text { for all } 1 \leq j \leq\left[t_{0}\right] .
\end{aligned}
$$

Therefore, by making use of 3.21 and $t /\left(2^{t}-1\right) \leq 1 / \log 2$ for all $t>0$, and using the condition $0<\delta \leq \mu-1$ with $\mu>1$, we see that

$$
\begin{aligned}
N_{1} & \leq C_{n}(n-r)^{-\frac{\mu+\delta}{\mu(\mu-\delta)}}\left(\sum_{j=1}^{\infty} 2^{-\frac{\mu(n-r)}{\mu-\delta} j}\right)^{1 / \mu} \\
& \leq C_{n}(n-r)^{-\frac{\mu+\delta}{\mu(\mu-\delta)}}\left(\sum_{j=1}^{\infty} 2^{-(n-r) j}\right)^{1 / \mu} \\
& =C_{n}(n-r)^{-\frac{\mu+\delta}{\mu(\mu-\delta)}}\left(\frac{1}{2^{n-r}-1}\right)^{1 / \mu} \\
& \leq C_{n}(n-r)^{-\frac{\mu+\delta}{\mu(\mu-\delta)}-\frac{1}{\mu}}=C_{n}(n-r)^{-\frac{2}{\mu-\delta}} .
\end{aligned}
$$

We next estimate $N_{2}$. Observe that the definition of $t_{0}$ implies

$$
\log (n-r)+t(n-r) \log 2 \geq \frac{t(n-r) \log 2}{1+\mu / \delta} \quad \text { for all } t \geq t_{0} .
$$


Since $t_{0}<\left[t_{0}\right]+1$, by using $(3.23)$ and $0<\delta \leq \mu-1, N_{2}$ can be estimated as follows:

$$
\begin{aligned}
N_{2} & \leq\left[\sum_{j=\left[t_{0}\right]+1}^{\infty}\left[\log \left((n-r) 2^{(n-r) j}\right)\right]^{-\frac{\mu}{\mu-\delta}}\right]^{1 / \mu} \\
& \leq\left[\sum_{j=\left[t_{0}\right]+1}^{\infty}\left[\frac{j(n-r) \log 2}{1+\mu / \delta}\right]^{-\frac{\mu}{\mu-\delta}}\right]^{1 / \mu} \\
& \leq C_{\delta}(n-r)^{-\frac{1}{\mu-\delta}}\left(\sum_{j=\left[t_{0}\right]+1}^{\infty} j^{-\frac{\mu}{\mu-\delta}}\right)^{1 / \mu} \\
& \leq C_{\delta}(n-r)^{\frac{1}{\mu-\delta}}\left(\int_{1}^{\infty} t^{-\frac{\mu}{\mu-\delta}} d t\right)^{1 / \mu} \leq C_{\delta}(n-r)^{-\frac{1}{\mu-\delta}} .
\end{aligned}
$$

Thus combining $(3.22)$ with $(3.24)$, we obtain

$$
\left[\sum_{j=1}^{\infty}\left[\log \left(1+(n-r) 2^{(n-r) j}\right)\right]^{-\frac{\mu}{\mu-\delta}}\right]^{1 / \mu} \leq C_{n, \delta}(n-r)^{-\frac{2}{\mu-\delta}} .
$$

CASE 2: $t_{0}<1$. In this case, the inequality $(3.23)$ is available for all $t=j \in \mathbb{N}$, and then, in the quite same manner as for $N_{2}$,

$$
\left[\sum_{j=1}^{\infty}\left[\log \left(1+(n-r) 2^{(n-r) j}\right)\right]^{-\frac{\mu}{\mu-\delta}}\right]^{1 / \mu} \leq C_{\delta}(n-r)^{-\frac{1}{\mu-\delta}} .
$$

To sum up, by (3.15), 3.17), 3.25) and (3.26), we have

$$
\begin{aligned}
\|u\|_{B_{\Phi_{0}, w_{r}}^{0, \nu}} & =\|\psi * u\|_{L_{\Phi_{0}, w_{r}}}+\left(\sum_{j=1}^{\infty}\left\|\varphi_{j} * u\right\|_{L_{\Phi_{0}, w_{r}}}^{\nu}\right)^{1 / \nu} \\
& \leq C_{n, \delta}\left(\frac{1}{n-r}\right)^{\frac{2}{\mu-\delta}}\left[\|\psi * u\|_{L_{p}}+\left(\sum_{j=1}^{\infty}\left(2^{\frac{n}{p} j}\left\|\varphi_{j} * u\right\|_{L_{p}}\right)^{\rho}\right)^{1 / \rho}\right] \\
& =C_{n, \delta}\left(\frac{1}{n-r}\right)^{\frac{2}{\mu-\delta}}\|u\|_{B_{p}^{n / p, \rho}},
\end{aligned}
$$

which is the desired estimate.

Acknowledgements. The author would like to express his gratitude to the referee for his/her valuable comments. In addition, the author is supported by the Fujukai Foundation.

\section{References}

[BL] J. Bergh and J. Löfström, Interpolation Spaces. An Introduction, Grundlehren Math. Wiss. 223, Springer, Berlin, 1976.

[B1] H. Q. Bui, Remark on the characterization of weighted Besov spaces via temperatures, Hiroshima Math. J. 24 (1994), 647-655. 
[B2] H. Q. Bui, Weighted Besov and Triebel spaces: Interpolation by the real method, ibid. 12 (1982), 581-605.

[B3] -, Weighted Young's inequality and convolution theorems on weighted Besov spaces, Math. Nachr. 170 (1994), 25-37.

[DHK] P. Drábek, H. Heinig and A. Kufner, Higher-dimensional Hardy inequality, in: General Inequalities 7, Int. Ser. Numer. Math. 123, Birkhäuser, Basel, 1997, $3-16$.

[HS] D. D. Haroske and L. Skrzypczak, Entropy and approximation numbers of embeddings of function spaces with Muckenhoupt weights I, Rev. Mat. Complut. 21 (2008), 135-177.

[KLSS1] Th. Kühn, H. G. Leopold, W. Sickel and L. Skrzypczak, Entropy numbers of embeddings of weighted Besov spaces II, Proc. Edinburgh Math. Soc. (2) 49 (2006), 331-359.

[KLSS2] -, 一, 一, 一, Entropy numbers of embeddings of weighted Besov spaces III. Weights of logarithmic type, Math. Z. 255 (2007), 1-15.

[M] B. Muckenhoupt, Weighted norm inequalities for the Hardy maximal function, Trans. Amer. Math. Soc. 165 (1972), 207-226.

[NW] S. Nagayasu and H. Wadade, Characterization of the critical Sobolev space on the optimal singularity at the origin, J. Funct. Anal. 258 (2010), 3725-3757.

[Og] T. Ogawa, A proof of Trudinger's inequality and its application to nonlinear Schrödinger equations, Nonlinear Anal. 14 (1990), no. 9, 765-769.

[OgOz] T. Ogawa and T. Ozawa, Trudinger type inequalities and uniqueness of weak solutions for the nonlinear Schrödinger mixed problem, J. Math. Anal. Appl. 155 (1991), 531-540.

[Oz] T. Ozawa, On critical cases of Sobolev's inequalities, J. Funct. Anal. 127 (1995), 259-269.

[R1] Y. Rakotondratsimba, Weighted $L^{p}-L^{q}$ inequalities for the fractional integral operator when $1<q<p<\infty$, SUT J. Math. 34 (1998), 209-222.

[R2] -, Weighted Young inequalities for convolutions, Southeast Asian Bull. Math. 26 (2002), 77-99.

[RaRe] M. M. Rao and Z. D. Ren, Theory of Orlicz Spaces, Monogr. Textbooks Pure Appl. Math. 146, Dekker, New York, 1991.

[T1] H. Triebel, Characterizations of Besov-Hardy-Sobolev spaces: a unified approach, J. Approx. Theory 52 (1988), 162-203.

[T2] -, Interpolation Theory, Function Spaces, Differential Operators, 2nd ed., Johann Ambrosius Barth, Heidelberg, 1995.

[T3] - Theory of Function Spaces III, Birkhäuser, Basel, 2006.

[W] H. Wadade, Remarks on the Gagliardo-Nirenberg type inequality in the Besov and the Triebel-Lizorkin spaces in the limiting case, J. Fourier Anal. Appl. 15 (2009), 857-870.

Hidemitsu Wadade

Advanced Mathematical Institute

Osaka City University

3-3-138 Sugimoto, Sumiyoshi-ku

558-8585 Osaka, Japan

E-mail: wadade@sci.osaka-cu.ac.jp, hidemitsuwadade@gmail.com 